\title{
Neumonitis por sales de oro en la artritis psoriásica: a propósito de dos casos
}

\author{
F. J. MANERO RUIZ, R. LARRAGA PALACIO*, I. HERRERO LABARGA* \\ M. FERRER PERALTA
}

Servicios de Reumatología y *Neumología. Hospital Miguel Servet. Zaragoza

\author{
PNEUMONITIS BY CHRYSOTHERAPY IN PSORIATIC ARTHRITIS: \\ TWO CASES REPORTS
}

\begin{abstract}
RESUMEN
Las sales de oro se utilizan para tratar la artritis reumatoide y la artritis psoriásica, generalmente son bien toleradas y cuando aparecen efectos secundarios suelen ser menores. La neumopatía por sales de oro es un efecto tóxico infrecuente que suele presentarse en los pacientes con artritis reumatoide, expresandose de tres formas diferentes: como neumonitis intersticial, bronquiolitis obliterante con neumonia organizada y bronquiolitis obliterante, siendo la primera su forma habitual de presentación. El diagnóstico se realiza por la clínica, radiología de tórax, el LBA, y la TAC torácica, practicando la biopsia pulmonar sólo en los casos que se quieran excluir otros procesos pulmonares. En la artritis psoriásica sólo hay publicados algunos casos de neumopatía por sales de oro. Se presentan dos pacientes con neumonitis por aurotiomalato que evolucionaron favorablemente después de suspender el tóxico y ser tratadas con corticoides.
\end{abstract}

PALABRAS CLAVE: Crisoterapia. Neumonitis por sales de oro. Artritis psoriásica. Toxicidad pulmonar.

\begin{abstract}
The gold-salts compounds have been used for the treatment of rheu matoid arthritis and of psoriatic artrhitis, they are generally tolerates and when have adverse effects they are minor. The gold-induced lung toxicity is a infrequent adverse effect of patients with rheumatoid arthri tis characterized by three types: interstitial penumonitis, bronchiolitis obliterans with organizing pneumonia and bronchiolitis obliterans, usually the first is the more frequent. The diagnostic is supported by the clinical manifestations, chest roentgenogram, bronchoalveolar lavage fluid and chest computed tomography scan. Biopsy study of the lung, is only needed for exclusion of other pulmonary disease. The gold-induced lung toxicity in the psoriatic artrhitis is rarelly described, we report two cases with gold-induced pneumonitis with benign clinical course after cessation of therapy and treatment with corticosteroids.
\end{abstract}

KEY WORDS: Chrysotherapy. Gold-induced pneumonitis. Psoriatic arthritis. Pulmonary toxicity.

Manero Ruiz FJ, Larraga Palacio R, Herrero Labarga I. Ferrer Peralta M. Neumonitis por sales de oro en la artritis psoriásica: a propósito de dos casos. An Med Interna (Madrid) 2002; 19: 237-240.

\section{INTRODUCCIÓN}

Desde 1920 se utiliza la crisoterapia por sus efectos inmunomoduladores en el tratamiento de la artritis reumatoide (AR) (1) y recientemente en la artritis psoriásica (2). Las sales de oro (SO) tienen con frecuencia efectos secundarios menores como dermatitis, estomatitis y discreta proteinuria. La toxicidad pulmonar por SO es poco frecuente y referida habitualmente al tratamiento de la AR. Más infrecuente todavia es la aparición de este efecto tóxico en la artritis psoriásica, donde se han descrito algunos casos (3-6). En una revisión de 110 artículos (7), donde se incluían 140 casos de neumopatía por sales de oro (NSO), 113 correspondieron a AR, sólo 1 a artritis psoriásica y el resto a tratamientos de pénfigo y asma. Dada la rareza de la NSO en la artritis psoriásica, aportamos dos pacientes con esta toxicidad pulmonar. La suspensión de las SO y el tratamiento con corticoides hizo que se resolviese totalmente el cuadro pulmonar.

\section{CASOS APORTADOS}

Caso 1: mujer de 54 años, no fumadora, sin contacto con animales domésticos ni otros antecedentes de interés. Había sido diagnosticada de poliartritis psoriásica, y desde hacía 4 meses estaba en brote que afectaba a las rodillas, tobillos y piés. Al no mejorar con AINEs, se inició tratamiento con aurotiomalato, $50 \mathrm{mg} / \mathrm{semana}$, salvo la $1^{\mathrm{a}} \mathrm{de}$ $10 \mathrm{mg}$ y la $2^{\mathrm{a}}$ de $25 \mathrm{mg}$. Cuando la dosis acumulada de aurotiomalato era de $250 \mathrm{mg}$, inició de forma progresiva: astenia, disnea de esfuerzo, tos seca, fiebre, náuseas, vómitos y lesiones eritemato-escamosas circinadas en zona anterior de tronco y hemiabdomen superior, sin

Trabajo aceptado: 17 de noviembre de 2000

Correspondencia: Fco. Javier Manero Ruiz. Corona de Aragón 18-20, 3 A. 50009 Zaragoza. Télf.: 9765553 15. e-mail: med008998@nacom.es. 
estomatitis, adenopatías ni visceromegalias. En la auscultación pulmonar había crepitantes bibasales. Analítica: Glucosa $132 \mathrm{mg} / \mathrm{dl}$; VSG: $3 \mathrm{~mm} 1^{\mathrm{a}}$ hora; leucocitos $16.200 / \mathrm{mm}^{3}, 88 \%$ neutrofilos, $0,05 \%$ eosinófilos (0-5\%); coagulación normal; inmunoglobulinas; complemento; factor reumatoide; serología de hepatitis B y C, virus respiratorios y bacterias atípicas; anticuerpos antinucleares, antitiroideos, antimitocondriales, antiDNAnativo; ENA: Ro,La, Sm, RNP; búsqueda de bacilo de Koch con cultivos, son negativos o normales. HLA: A2,A30, B51, B13, Cw6. PaO2: 68 mmHg; Pruebas funcionales respiratorias: déficit ventilatorio restrictivo leve con disminución TLC $77 \%$ con descenso moderado de la difusión, DLCO $54 \%$ y KCO $58 \%$. Radiografía de tórax: afectación intersticial difusa de predominio en campos pulmonares medios e inferiores (Fig. 1). TAC torácica: patrón reticular difuso con focos de afectación alveolar parcheada (Fig. 2). lavado broncoalveolar (LBA): alveolitis linfocitaria y cociente CD4/CD8: 0,07\% (1,50-2,00), no se detectan linfocitos B. Se suspendieron las sales de oro y fue tratada con $60 \mathrm{mg} / \mathrm{día}$ de prednisona, con pauta de reducción según la respuesta. A los 3 meses se encontraba asintomática y a los 6 meses la espirometria y la radiografía de tórax fueron normales. tres años después, la paciente permanecía asintomática.

Caso 2: mujer de 62 años de edad, sin hábitos tóxicos conocidos ni antecedentes de interés. Diagnosticada de psoriasis en codos y rodillas, presentaba desde hacía 6 años brotes de poliartritis psoriásica que controlaba con AINEs. En el último episodio articular ante la falta de mejoría con AINE se añadió aurotiomalato, $50 \mathrm{mg} / \mathrm{semana}$, salvo la $1^{\mathrm{a}}$ de $10 \mathrm{mg}$ y la $2^{\mathrm{a}}$ de $25 \mathrm{mg}$. Cuando había acumulado una dosis total de $135 \mathrm{mg}$ apareció un exantema pruriginoso en cuello, tronco y EEII, y de forma progresiva disnea de esfuerzo, tos seca, febrícula y malestar general. Analítica: LDH $471 \mathrm{UI} / \mathrm{L}$ (50-460); VSG $6 \mathrm{~mm} / 1^{\mathrm{a}}$ hora; eosinófilos 11,90\% (0-5\%); resto de hemograma y sistemático que incluye función hepática y renal; $\operatorname{IgE} 10(0-40)$, resto de inmunoglobulinas; complemento; anticuerpos antinucleares, antiDNAnativo, antitiroideos; serología para virus respiratorios y bacterias atípicas; búsqueda de bacilo de Koch con cultivos; normales o negativos. HLA A2, A3, B38, B44, Cw6, Dr4, Dr7. LBA: Alveolitis eosinofilíca (40\% de eosinofilos), cociente CD4/CD8: 0,07\% $(1,50-2,00)$. Pruebas funcionales respiratorias con disminución de volumen residual del $72 \%$, descenso de DLCO $62 \%$ y KCO $63 \%$. PaO2: 69 mmHg. Radiografía de tórax: ensanchamiento mediastínico anterosuperior compatible con adenopatias paratraqueales y patrón reticular basal. TAC torácica: Infiltrados alveolares en ambos
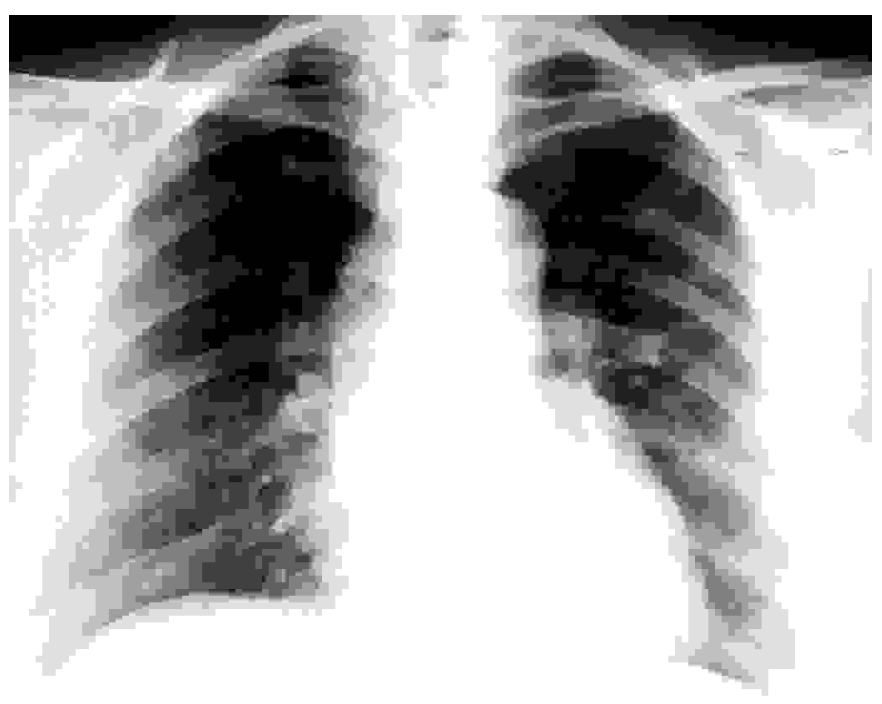

Fig. 1. Rx tórax: Patrón intersticial difuso de predominio en campos pulmonares medios e inferiores.

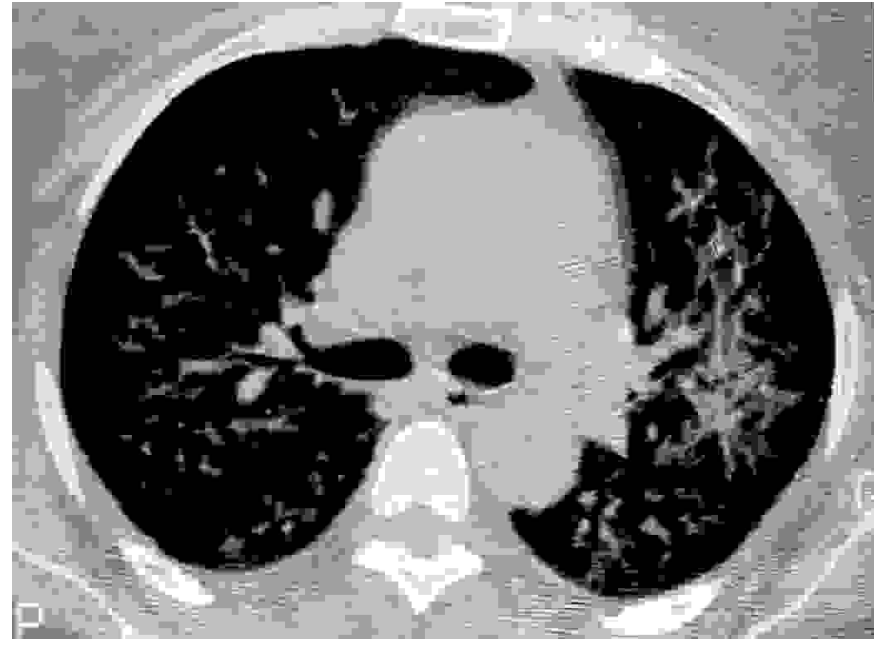

Fig. 2. TAC torácica: Patrón reticular difuso con focos de afectación alveolar parcheada.

lóbulos inferiores y lóbulo medio. Adenopatias en región paratraqueal y subcarínicas. Biopsia Transbronquial: mínimos signos inflamatorios. Se practicó mediastinoscopia y biopsia de dos pequeñas adenopatias con anatomía patológica de adenitis reactiva. Se suspendió el aurotiomalato y fue tratada con $40 \mathrm{mg}$ de prednisona/día que se disminuyó progresivamente hasta su retirada, con mejoría progresiva del cuadro respiratorio y desaparición de las lesiones dérmicas. Antes de los 6 meses la paciente estaba asintomática y las pruebas de función pulmonar y Radiografía de tórax eran normales.

\section{DISCUSIÓN}

Un volumen considerable de drogas, entre las que se incluyen los AINEs y las SO, se han asociado a toxicidad pulmonar (8). Los AINEs suelen provocar broncoespasmo y edema pulmonar, mucho más raro es la existencia de neumonitis por hipersensibilidad (1). Las dos pacientes habían recibido previamente AINEs sin presentar toxicidad pulmonar. Al poco tiempo de estar en tratamiento con aurotiomalato es cuando aparece una neumonitis sin existir patologia pulmonar previa y habiéndose descartado proceso infeccioso. La NSO es poco común y ha sido descrita sobretodo en mujeres con $\mathrm{AR}$, en cualquier momento del tratamiento, aunque sea preferente en los 3 primeros meses (1), y con dosis acumuladas que oscilan de $100 \mathrm{mg}$ a $3000 \mathrm{mg}$ (7). Aparece con cualquier vía de administración, oral (auranofín) $(9,10)$ o inyectable. De las dos formas inyectables de SO, aurotiomalato y aurotioglucosa, es el aurotiomalato el que provoca mas efectos secundarios, quizás relacionado con una absorción mas rápida.

Las SO pueden provocar tres formas diferentes de neumopatía: neumonitis intersticial, bronquiolitis obliterante con neumonia organizada y bronquiolitis obliterante. En nuestro primer caso la historia clínica, la radiología y la TAC torácica fueron sugestivas de bronquiolitis obliterante con neumonia organizada, pero no se realizó biopsia pulmonar para poder confirmarlo. El segundo caso es una neumonitis intersticial en la forma que asocia infiltrados eosinofílicos pulmonares (8). La neumonitis intersticial es la NSO más frecuente, en la tabla I se describen las características de 6 casos de toxicidad por sales de oro en la artritis psoriásica, nuestros dos casos y cua- 
tro recogidos de la bibliografía, donde sólo uno fue una bronquiolitis obliterante (5). Clínicamente la neumonitis intersticial se instaura de una forma aguda o progresiva con tos seca, fiebre, disnea, y exantema como manifestación extrapulmonar más frecuente (7). En sangre periférica hay eosinofilia en el $36 \%$ de los casos, como ocurría en la segunda paciente, asociándose esta circustancia a un buen pronóstico (7). En la radiografía de tórax aparece un infiltrado difuso intersticial o alveolar. Hay hipoxemia y en los estudios de función pulmonar un patrón ventilatorio restrictivo, destacando la alteración de la capacidad de difusión del monóxido de carbono (DLCO). El tratamiento consiste en la suspensión del fármaco, medidas generales y la utilización de corticoides a dosis de $0,5-1 \mathrm{mg} / \mathrm{kg} /$ día. con reducción progresiva de la dosis según la respuesta. La mayoria de los pacientes tienen una buena evolución con resolución completa del cuadro pulmonar.

La bronquiolitis obliterante con neumonia organizada y la bronquiolitis obliterante asociadas a SO son poco frecuentes. Cuando aparecen en pacientes con AR seropositiva no queda claro si es la propia AR la causante (1). No obstante hay un caso descrito de bronquiolitis obliterante en un paciente con artritis psoriásica que era factor reumatoide negativo (5), por lo que las SO pueden jugar un papel en esta forma de toxicidad pulmonar. La bronquiolitis obliterante con neumonia organizada se caracteriza por infiltrados alveolointersticiales bilaterales de forma parcheada y ocasionalmente lineares o nodulares (11). El tratamiento utilizado son los corticoides, con un pronóstico generalmente bueno (3), aunque ha habido algún caso que ha precisado ventilación mecánica (12). La bronquiolitis obliterante tiene peor pronóstico por su mala respuesta al tratamiento con corticoides, tanto en la AR (13) como en la artritis psoriásica (5).
En el LBA de las neumonitis inducidas por drogas, incluidas las SO (14) hay inversión del cociente CD4/CD8 (menor de $1,00 \%$ ) y un predominio linfocítico. Mas raro es que predominen los neutrófilos o eosinófilos como ocurría en nuestro segundo caso, donde había un notable incremento de eosinófilos, coincidiendo con la eosinofilia en sangre perférica. El LBA de la NSO es diferente del encontrado en la enfermedad pulmonar reumatoide donde hay un predominio de neutrófilos con inversión del cociente CD4/CD8, y en los casos donde predominan los linfocitos el cociente CD4/CD8 está incrementado (15).

A diferencia de la artritis psoriásica, la AR tiene una patología pulmonar propia que es coincidente con la que aparece en la NSO (15), lo que puede crearnos problemas diagnósticos. Para ayudar al diagnóstico diferencial con la enfermedad pulmonar de la AR, Tomioka ha establecido unos criterios (7), destacando como sugestivos de neumonitis intersticial por SO: el predominio femenino, la fiebre, el rash cutáneo, la ausencia de nódulos subcutáneos o dedos en palillo de tambor, los bajos títulos de factor reumatoide, la linfocitosis en el LBA y la TAC torácica con infiltrados alveolares peribroncovasculares.

La toxicidad pulmonar por agentes químicos puede ser directa, como la provocada por la amiodarona, o indirecta de origen inmunológico como en las sales de oro (16). Se sugiere que el mecanismo inmunológico es por una reacción de hipersensibilidad, dado el predominio linfocitario en el LBA, la eosinofilia periférica, y la buena respuesta a corticoides (1). También existiría una predisposición genética, con mayor asociación a determinadas moléculas HLA. Así la proteinuria se asocia con mayor frecuencia al HLA DR3 $(17,18)$ y las manifestaciones mucocutáneas al HLA

TABLA I

CARACTERÍSTICAS DE 6 PACIENTES CON ARTRITIS PSO RIÁSICA Y TOXICIDAD PULM O NAR PO R AURO TIO M ALATO

\begin{tabular}{|c|c|c|c|c|c|c|}
\hline & Caso 1 & Caso 2 & Fernández (3) & Ballesteros (4) & Schwartzman (5) & Sabadell (6) \\
\hline$\overline{\text { Edad* }}$ & 54 & 62 & 67 & 43 & 41 & 39 \\
\hline Sexo & Mujer & M ujer & M ujer & Hombre & Hombre & Hombre \\
\hline Dosis total & $250 \mathrm{mg}$ & $135 \mathrm{mg}$ & $600 \mathrm{mg}$ & $735 \mathrm{mg}$ & $595 \mathrm{mg}$ & $585 \mathrm{mg}$ \\
\hline Curso & Progresivo & Progresivo & Agudo & Progresivo & Progresivo & Progresivo \\
\hline Tos seca & Sí & Sí & Sí & Sí & Sí & No \\
\hline Disnea & Sí & Sí & Sí & Sí & Sí & Sí \\
\hline Fiebre & Sí & Sí & Sí & No & No & No \\
\hline Lesiones de piél & Eritemato-escamosas & Exantema & Exantema & No & No & No \\
\hline Crepitantes & Sí & NR & Sí & Sí & NR & NR \\
\hline DLCO & $54 \%$ & $62 \%$ & $50 \%$ & NR & NR & NR \\
\hline Rx tórax & Intersticial & Intersticial & Intersticial & Intersticial & no significativa & Intersticial \\
\hline
\end{tabular}

LBA:

\begin{tabular}{|c|c|c|c|c|c|c|}
\hline $\begin{array}{l}\text { Predominio: } \\
\text { Cociente }\end{array}$ & Linfocitos & Eosinófilos & NR & NR & Linfocitos & NR \\
\hline CD4/CD8: & Invertido & Invertido & NR & NR & Invertido & NR \\
\hline BTB & NR & NR & NR & Inespecífica & NR & NR \\
\hline Corticoides & 60 mg/ día & 40 mg/día & $1 \mathrm{mg} / \mathrm{kg} / \mathrm{día}$ & $1 \mathrm{mg} / \mathrm{kg} / \mathrm{día}$ & 40 mg/día & $1 \mathrm{mg} / \mathrm{kg} / \mathrm{dí} a$ \\
\hline total & Sí & Sí & Incompleta & Sí & No & Sí \\
\hline
\end{tabular}

*Edad en años; NR: no realizado; DLCO : capacidad de difusión del monóxido de carbono; BO: bronquiolitis obliterante; LBA: lavado broncoalveolar, BTB: biopsia transbronquial. 
DR5 (19). En la toxicidad pulmonar se ha encontrado asociado el fenotipo HLA A3 B35 Dw1 BfF C4A3,2 (BO), y el HLA B40 (18) sobretodo cuando se añade un alelo nulo C4 (20).

La NSO es una complicación descrita generalmente en la AR y raramente en la artritis psoriásica. Es un proceso que tie- ne buena evolución cuando se suspende el tóxico, por lo que su sospecha en la artritis psoriásica evitará exploraciones y tratamientos innecesarios. Su diagnóstico se basará en la clínica, radiografía de tórax, el LBA, y la TAC torácica, realizando la biopsia pulmonar sólo en los casos que sea preciso excluir otros procesos pulmonares.

\section{Bibliografía}

1. Libby D, White DA. Pulmonary toxicitiy of drugs used to treat systemic autoimmune diseases. Clin Chest Med 1998; 19: 809-21.

2. Richter MB, Kinsella P, Corbet M. Gold in psoriatic arthropathy. Ann Rheum Dis 1980; 39: 279-80.

3. Fernández Álvarez R, Dominguez del Álamo M J, Molinos Martín L, Babio Herráez J. Toxicidad pulmonar asociada a tratamiento con sales de oro: experiencia con 4 casos. Med Clin (Barc) 1994; 102: 475.

4. Ballesteros JA, Maimó A,Usandizaga I, Orfila B. Toxicidad pulmonar asociada a tratamiento con sales de oro. Med Clin (Barc) 1995; 104: 44-5.

5. Schwartzman KJ, Bowie DM, Yeadon C, Fraser R, Sutton DE, Levy RD. Constrictive bronchiolitis obliterans following gold therapy for psoriatic arthritis. Eur Respir J 1995; 8: 2191-3.

6. Sabadell C, Casado E, Izquierdo J, Olivé A. Neumonitis por sales de oro en la artritis psoriásica. Rev Esp Reumatol 1999; 26: 39-40.

7. Tomioka H, Talmadge E, King Jr. Gold-induced pulmonary disease: Clinical features, outcome, and differentiation from rheumatoid lung disease. Am J Respir Crit Care Med 1997; 155: 1011-20.

8. Foucher P, Biour M, Blayac JP et al. Drugs that may injure the respiratory system. Eur Respir J 1997; 10: 265-79.

9. Abeledo C, Jolis R, Jaén A, Hernandez L. Bronquiolitis obliterante con neumonía organizada secundaria a sales de oro. Med Clin (Barc) 1993; 101: $42-3$.

10. O’Duffy JD, Luthra HS, Unni KK, Hyatt RE. Bronchiolitis in a rheumatoid arthritis patient receiving auranofin. Arthritis Rheum 1986; 29: 556-9.

11. Epler GR. Bronchiolitis obliterans organizing pneumonia: Definition and clinical features. Chest 102 (suppl): 3S-6S.

12. Blancas R, Moreno JL, Martín F, de la Casa R, Oñoro JJ,Gomez V et al. Alveolar-intersticial pneumopathy gold-salts compounds administration, requiring mechanical ventilation. Intens Care Med 1998; 24: 1110-2.

13. Holness L, Tennenbaum J, Cooter NBE, Grossman RF. Fatal bronchiolitis obliterans associated with chrysotherapy. Ann Rheum Dis 1983; 42: 593-6.

14. Akoun GM, Cadranel JL, Milleron BJ, Flammang MP, Mayaud ChM Bronchoalveolar lavage cell data in 19 patients with drugs-associated pneumonitis (except amiodarone). Chest 1991; 99: 98-104.

15. Anaya JM, Diethelm L, Ortiz LA, Gutierrez M, Citera G, Welsh RA et al. Pulmonary involvement in rheumatoid arthritis. Semin Arthritis Rheum 1995; 24: 242-54.

16. Martin WJ. Pulmonary toxicity induced by chemical agents. Eur Respir J 1990; 3: 375-6.

17. Sakkas LI, Chikanza IC, Vaughan RW, Welsh KI, Panayi GS. Gold induced nephropathy in rheumatoid arthritis. Ann Rheum Dis 1993; 52: 300-1.

18. Hakala M, van Assendelft AHW, Ilonen J, Jalava S, Tiilikainen A. Association of different HLA antigens with various toxic effects of gold salts in rheumatoid arthritis. Ann Rheum Dis 1986; 45:177-182.

19. Rodriguez Pérez M, Gonzalez Dominguez J, Matarán L, García Pérez S, Salvatierra D. Association of HLA-DR5 with mucocutaneous lesions in patients with reumatoid arthritis receiving gold sodium thiomalate. J Rheumatol 1994; 21: 41-3.

20. Partanen J, van Assendelft AHW, Koskimies S, Forsberg S, Hakala M, , Ilonen J. Patients with rheumatoid arthritis and gold-induced pneumonitis express two high-risk major histocompatibility complex patterns. Chest 1987; 92: 277-81. 